\title{
Assessing performance of the MERGE model for simulating gully interventions
}

\author{
M. J. Prentice ${ }^{\text {a }(D), ~ M . ~ W . ~ W a u d ~}{ }^{\text {b }}$ (D), D. P. Hamilton ${ }^{a}$ (iD), and M. E. Roberts ${ }^{\text {ac }}$ \\ ${ }^{a}$ Australian Rivers Institute, Griffith University, 170 Kessels Road, Nathan, Queensland 4111, Australia \\ ${ }^{\mathrm{b}}$ Healthy Land and Water, Brisbane City, Queensland 4000, Australia \\ ${ }^{\mathrm{c}}$ School of Environment and Science, Griffith University, Queensland 4111, Australia
}

Email: m.prentice@griffith.edu.au

\begin{abstract}
Gully erosion is a significant s afety, e conomic, and environmental issue which affects agricultural productivity, infrastructure stability, and water quality of receiving waters. Hot spots of gully erosion have been identified across every continent and control and mitigation methods are s ought. Despite a variety of interventions available to prevent gully formation and to rehabilitate existing gullies, cost-effective interventions need to be tailored for each gully. MERGE (modelling erosion resistance for gully erosion) is a one-dimensional process-based model for gully erosion, designed to simulate erosion events in classical gullies and quantify the benefit of interventions in s i lico. Simulations a re u sed to inform the a ppropriateness of interventions applied in situ. The aim of this study was to assess the performance of MERGE at assessing the benefits of three commonly applied gully treatment interventions using on-ground input data.
\end{abstract}

The model was run under steady state conditions with input data specific to Fe rnvale Gu lly in the Lower Brisbane River Catchment, South-East Queensland, Australia. Interventions simulated included; reducing the flow $(Q)$ at the gully head (as a result of catchment w orks), increasing vegetation cover of the gully channel, and rock-capping of the gully head. Interventions relating to reducing $Q$ and increasing vegetation cover were carried out for 'small', 'medium', 'large', and 'very-large' modifications to $Q$ and Manning's roughness $(n)$, respectively. The intervention relating to rock-capping was carried out by assuming no erosion in the gully head. Interventions were also simulated for combinations of 'small' reduction in $Q$, 'small' reduction in vegetation cover, and rock-capping. For each intervention, erosion resistance $(J)$-being a largely unknown quantity in the scientific literature-was input as a selection of values ranging between high and low erodibility.

MERGE was demonstrated to be a useful tool to guide on-ground decision making for gully management interventions. Specifically, the s imulations p rovide i nsight i nto the r elative b enefits of ea ch in tervention onground at Fernvale Gully. MERGE demonstrated that each gully intervention performed markedly differently depending on antecedent gully conditions. MERGE also demonstrated that reducing $Q$ resulted in a proportional linear reduction in sediment flux $(Q C)$ exiting the gully, whereas increasing vegetation cover resulted in a non-linear reduction in $Q C$, with ever increasing vegetation cover producing reduced returns. The benefit of combining interventions was not additive, illustrating the benefit of MERGE for assessing potential outcomes when more than one intervention is to be implemented.

The implementation of MERGE in this study allowed the most effective intervention strategies to be identified on-ground for the gully of interest, thus demonstrating the potential of MERGE to guide decision making for gully management. Therefore, future application of MERGE might look to apply scenarios to help inform the most appropriate interventions across a suite of gullies. Such an assessment might entail gullies with complex morphology (i.e., dendritically shaped, and multiple heads), and a broader scope of interventions than considered here (e.g., porous check dams, and the application of gypsum and fertilisers).

Keywords: Erosion, gullies, intervention, process-based model, sediment 


\section{INTRODUCTION}

Gully erosion-suspension and export of sediment from gullies due to catchment runoff-is a significant safety, economic, and environmental issue (Bartley et al. 2020, Roberts 2020). Safety and economic implications of gully erosion often relate to limitation of agricultural productivity, and destabilisation of landscapes, housing, and other essential infrastructure. Environmental implications typically relate to the export of sediment and nutrients to the riverine, lacustrine, and marine environments. Elevated sediment and nutrients loads result in the deterioration of water quality as a result of high turbidity, increased light attenuation, and nutrient regimes that fuel toxic algal blooms (Hamilton et al. 2016). Further, with hot spots of gully erosion having been identified across every continent, control and mitigation of gully erosion is therefore important for environmental protection on a global scale (Castillo \& Gómez 2016).

The impact of gullies and their erosion on agricultural productivity, infrastructure, and water quality has motivated extensive efforts to prevent gullying and rehabilitate existing gullies (Roberts et al. submitted, Bartley et al. 2020). A variety of interventions exist, that function by reducing the volume (or velocity) of the flow $(Q)$ entering the gully (e.g., by increasing catchment vegetation cover), or increasing the erosion resistance ( $J$; aka, the sediment cohesion factor) within the gully (e.g., by rock-capping of the gully head, or increasing gully vegetation cover; Wilkinson et al. 2019, Bartley et al. 2020). These interventions vary markedly in their efficacy and cost-effectiveness, with many of most efficacious interventions (e.g., rock-capping of the gully head) involving extensive, costly engineering works. By contrast, many of the cheapest interventions (e.g., increasing vegetation cover) are less likely to offer a significant benefit in all instances. Consequently, choosing the most appropriate intervention is critical to ensure a cost-effective treatment that delivers on environmental targets.

Models of gully erosion have been developed to predict gully growth, quantify the contributions of gullies to the water quality of receiving water bodies, and to support and inform rehabilitation efforts (Roberts et al. submitted). Despite this, existing models of gully erosion are predominantly empirical in nature. These models have limitations for quantifying the costs and benefits of different interventions (Roberts 2020). Process-based models, in contrast, are better equipped to handle analysis of the potential benefits of different interventions owing to quantitative aspects of their formulation (Roberts et al. submitted). As such, process-based models have been identified as necessary to support catchment management (Prosser 2018, Roberts 2020), and more broadly to ecological systems in a changing world (Cuddington et al. 2013).

MERGE (modelling erosion resistance for gully erosion; Roberts 2020 [an extension of Roberts 2019]) is a process-based model for gully erosion that has been designed to capture the physical interactions of erosion events in classical gullies and explore the benefit of different interventions for their potential implementation in situ (Roberts 2020). MERGE replicates the important elements of gully erosion; including attainment of a steady concentration under constant conditions, development of a depositional layer; and in the dynamic case, first flush effects and hysteresis (Roberts 2020). The analytical steady-state solutions have also been demonstrated as excellent approximations to the full dynamic solutions (Roberts 2020).

The aim of this study was to evaluate the performance of MERGE to assess the benefits of management interventions on-ground in a classical gully. The aim was addressed by quantifying the sediment flux, $Q C$, exiting the gully in response to three different interventions relating to: (1) reducing $Q$ entering the gully head (as a result of catchment works), (2) vegetating the gully channel, and (3) rock-capping the gully head.

\section{METHODS}

Evaluation of the MERGE models performance to assess different interventions was determined using data collected from Fernvale Gully.

MERGE Model description. MERGE (Roberts 2020) is an event-based, one-dimensional model for gully erosion. It uses conservation of mass within the water column subject to erosion (source) and deposition (sink) to calculate the flux of sediment exiting a gully. MERGE captures erosion due to two key processes: (1) headcut retreat due to the action of runoff entering the gully as a waterfall at the gully head, and (2) scouring of the floor and walls throughout the gully due to channel flow. Deposition results from sediment settling out of the water column; and this deposited sediment is available for re-entrainment.

Analytical steady state solutions to MERGE (Roberts 2020, Eqns 12-15) are used in the present study to describe the sediment concentration along the gully and the sediment flux exiting the gully. These analytical solutions capture two cases: (1) where recently deposited sediment forms a depositional layer on the gully 
floor, protecting the original soil matrix from erosion; and (2) where the rate of erosion exceeds the rate of deposition and no layer is formed. Key parameters in MERGE are the mean concentration of sediment within the water column $(C), Q, J$, and Manning's roughness $(n)$. For a complete list of the model parameters and values adopted for this study refer to Table 1, and for a complete description of MERGE refer to Roberts (2020).

Table 1. Fernvale Gully baseline parameters and input values

\begin{tabular}{|c|c|c|c|}
\hline Parameter & Symbol & Value & Unit \\
\hline Carrying capacity & $C^{*}$ & $0.2 \sigma$ & $\mathrm{kg} \mathrm{m}^{-3}$ \\
\hline Concentration gradient & $b$ & 1 & Unitless \\
\hline Depth of gully & $D_{0}$ & 2 & $\mathrm{~m}$ \\
\hline Depth of gully water & $d$ & 0.584 & $\mathrm{~m}$ \\
\hline Dynamic viscosity of water & $\mu$ & $1.0031 \times 10^{-6} \rho$ & $\mathrm{kg} \mathrm{m}^{-1} \mathrm{~s}^{-1}$ \\
\hline Erosion resistance & $J$ & $0-3200$ & $\mathrm{~W} \mathrm{~s} \mathrm{~kg}^{-1}$ \\
\hline Flow & $Q$ & N/A & $\mathrm{m}^{3} \mathrm{~s}^{-1}$ \\
\hline Fluid density & $\rho$ & 1000 & $\mathrm{~kg} \mathrm{~m}^{-3}$ \\
\hline Friction term & $F$ & 0 & $\mathrm{~W} \mathrm{~s} \mathrm{~kg}^{-1}$ \\
\hline Gravity & $g$ & 9.81 & $\mathrm{~m} \mathrm{~s}^{-2}$ \\
\hline Initial concentration & $C_{0}$ & 0 & $\mathrm{~kg} \mathrm{~m}^{-3}$ \\
\hline Length of gully & $L$ & 170 & $\mathrm{~m}$ \\
\hline Length of gully head & $L_{h}$ & 1 & $\mathrm{~m}$ \\
\hline Manning's roughness & $n$ & 0.027 & $\mathrm{~s} \mathrm{~m}^{-1 / 3}$ \\
\hline Power proportion & $k$ & 0.2 & Unitless \\
\hline Sediment density & $\sigma$ & 1330 & $\mathrm{~kg} \mathrm{~m}^{-3}$ \\
\hline Sediment size & $R$ & $1.6 \times 10^{-5}$ & $\mathrm{~m}$ \\
\hline Settling velocity & $w_{s}$ & 0.05 & $\mathrm{~m} \mathrm{~s}^{-1}$ \\
\hline Slope of gully & $S$ & 0.012 & Unitless \\
\hline Width of gully & $W$ & 5.5 & $\mathrm{~m}$ \\
\hline
\end{tabular}

Study Site description. Fernvale Gully (Figure 1) is a classical gully located in the Lower Brisbane River Catchment, South-East Queensland, Australia. Its catchment is 95 ha, with the upper third of its catchment mostly urban development and the lower two-thirds mostly agricultural land used for cattle grazing. Fernvale Gully measures $170 \mathrm{~m}$ from its primary head to its outfall. The gully has a slope $(S)$ of $1.2 \%$, and Manning's roughness $(n)$ between 0.027 and $0.050 \mathrm{~s} \mathrm{~m}^{-1 / 3}$ depending on recency of cattle grazing. The gully is intermittently vegetated, and although grass as high as 1-2 m has been observed (as shown in Figure 1 j, c) its default state is bare sediment. The outfall is into the Lower Brisbane River. Management and mitigation of poor water quality in the Lower Brisbane River is critical to the quality of water supply from the river to Mount Crosby Water Treatment Plant, and the health of Moreton Bay, which includes an internationally important (Ramsar) wetland.

MERGE Model assessment. The MERGE model was compiled and implemented in Python v 3.9.6 (Python 2021). Model parameter values (Table 1) were determined from measured values collected in Fernvale Gully, and literature values identified in Roberts (2020). Measured values collected from Fernvale Gully included; depth of the gully $\left(D_{0}\right)$, depth of the gully water $(d)$, length of the gully $(L)$, length of the gully head $\left(L_{h}\right), n, S$, and width of the gully $(W)$. Measured values quantified in Fernvale Gully were determined using conventional techniques by Healthy Land and Water (HLW). $d$ was determined using Manning's equation by relating $d$ to $Q$ for a 1 in 50 year event at $8.0 \mathrm{~m}^{3} \mathrm{~s}^{-1}$. $n$ was input-per worst case scenario (i.e., post cattle grazing) —at 0.027 $\mathrm{s} \mathrm{m}^{-1 / 3}$. J-being an largely unknown quantity in the scientific literature-was input as a selection of values between $0 \mathrm{~W} \mathrm{~s} \mathrm{~kg}^{-1}$ (i.e., low $J$, equivalent to high erodibility) and $3200 \mathrm{~W} \mathrm{~s} \mathrm{~kg}^{-1}$ (i.e., high $J$, equivalent to low erodibility). Aside from changes to the intervention parameter of interest, all other values were input per Table 1. 

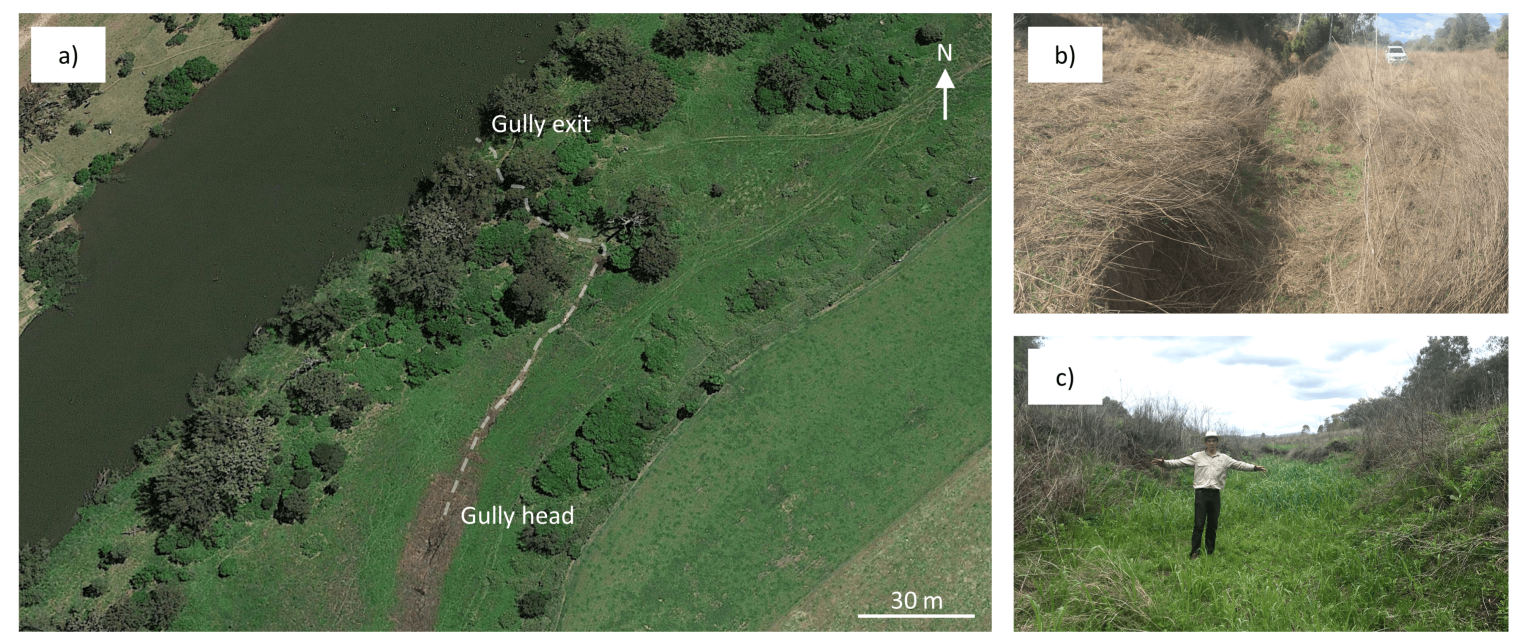

Figure 1. Fernvale gully study site, showing: a) gully and surroundings (GoogleEarth), b) down-gully at the gully head (Healthy Land and Water), and c) up-gully near the gully exit (Healthy Land and Water).

To evaluate the performance of MERGE in assessing the benefits of management interventions, we explored the effect of three different interventions on the $Q C$ exiting the gully. Intervention \#1 reduced $Q$ entering the gully head (due to catchment works) by $20,40,60$, and $80 \%$ of $8 \mathrm{~m}^{3} \mathrm{~s}^{-1}$, representing 'small', 'medium', 'large', and 'very-large' interventions, respectively. $Q$ was determined from Manning's equation relating $d$ to $Q$. Due to the non-linearity of Manning's equation, we set $d$ directly, and $Q$ was calculated in the model. Intervention \#2 increased vegetation cover in the gully from 0 to $20,40,60$, and $80 \%$, representing 'small', 'medium', 'large', and 'very-large' interventions, respectively. The effect of increased vegetation cover was modelled via $n$ using Roberts (2020, Eqn 16), which assumes a vegetation height of $20 \mathrm{~cm}$. Increasing vegetation cover results in increasing $n$ and hence decreases $Q$ for a given flow depth $d$. $d$ was assumed not to change with $n$, although we acknowledge the true situation is more complicated. Intervention \#3 was rockcapping the gully head where it was assumed that no erosion occurred within the gully head, and thus only channel erosion was considered in these scenarios.

As interventions are rarely applied individually, the ability of MERGE to access multiple concurrent interventions was considered by pairing interventions from a 'small' reduction in $Q$, 'small' increase in vegetation cover, and rock capping. Consideration was also given to all three interventions applied concurrently.

\section{RESULTS}

Erosion resistance. Increasing $J$ resulted in a reduction in $Q C$ in Fernvale Gully, both along the gully (i.e., the head and channel) and exiting the gully (Figure 2 ). In the most erosive case, $J=0$, maximal sediment transport, that is the concentration carrying capacity $\left(C^{*}\right)$, was achieved within a few metres. All other $J$ values considered, ranging from $6.25 \mathrm{~W} \mathrm{~s} \mathrm{~kg}^{-1}$ to $3200 \mathrm{~W} \mathrm{~s} \mathrm{~kg}^{-1}$ stayed below the carrying capacity, resulting in sediment fluxes at the gully exit between 3766.2 and $19.8 \mathrm{~kg} \mathrm{~s}^{-1}$, respectively.

Intervention 1: Reducing the flow entering at the gully head. Decreasing $Q$ entering the gully head resulted in a linear reduction in $Q C$ at the gully exit (Figure $3 \mathfrak{z}$ ).

Intervention 2: Increasing vegetation cover in the gully channel. Increasing vegetation cover in the gully channel resulted in a nonlinear reduction in $Q C$ exiting the gully (Figure 3 3 ). More specifically, 'small', 'medium', 'large', and 'very-large' increases in vegetation cover resulted in reductions of $Q C$ exiting the gully of $57,72,80$, and $84 \%$, respectively.

Intervention 3: Rock-capping the gully head. Rock-capping the gully head gave a 23, 43, 48, 49, and $50 \%$, reduction in $Q C$ exiting the gully, at $J$ values of 6.25, 25, 100, 400, and 1600, respectively (Figure 3a).

Intervention combinations. Combinations of interventions did not have an additive effect at reducing $Q C$ exiting the gully (Figure 4). Of the three paired combinations, increasing vegetation cover and rock-capping 
Prentice et al., Assessing performance of the MERGE model for simulating gully interventions

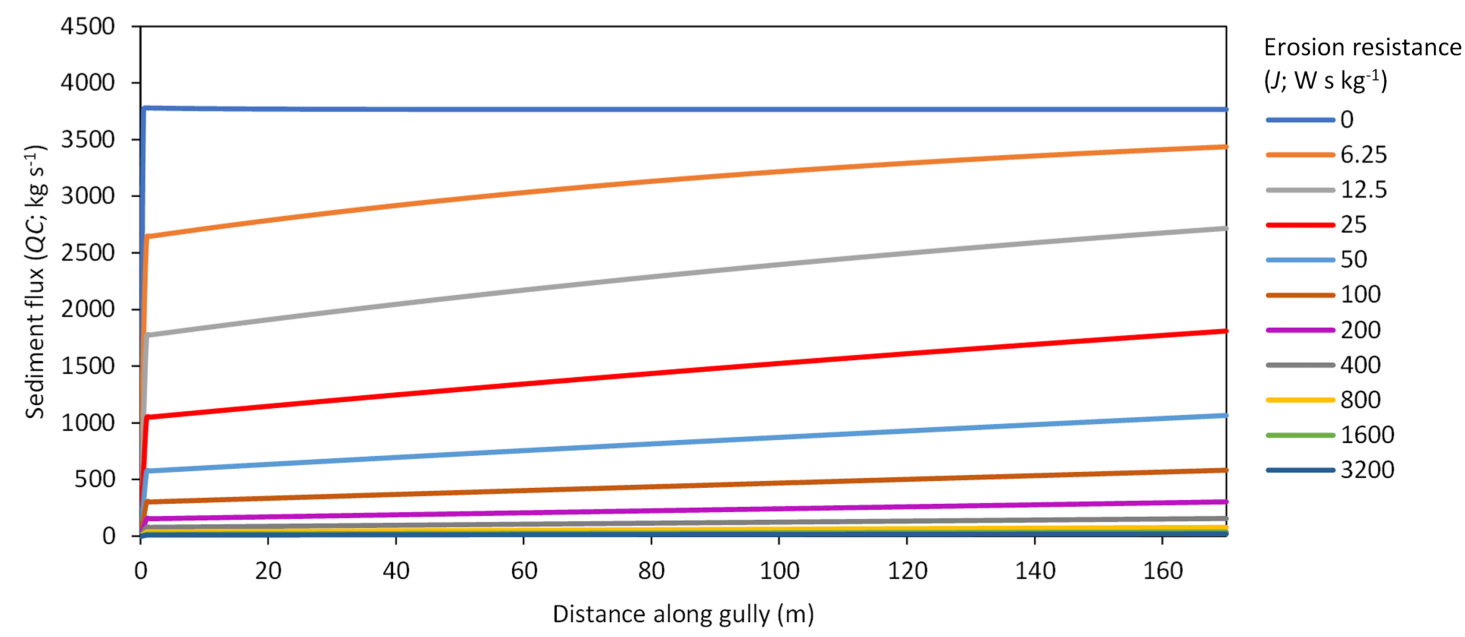

Figure 2. Sediment flux $\left(Q C ; \mathrm{kg} \mathrm{s}^{-1}\right)$ along the gully for a selection of erosion resistance $(J)$ values between 0 and $3200 \mathrm{~W} \mathrm{~s} \mathrm{~kg}^{-1}$.
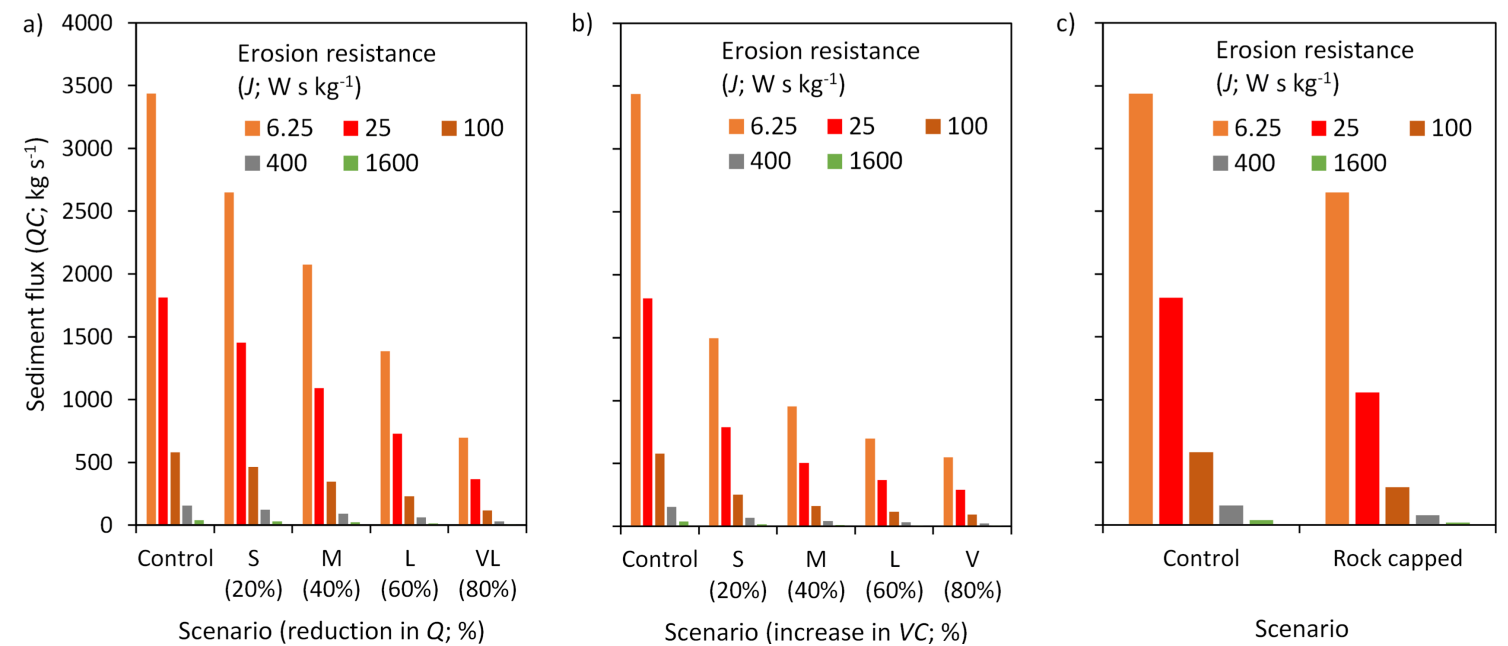

Figure 3. The effect of interventions $1-3$ on the sediment flux $\left(Q C ; \mathrm{kg} \mathrm{s}^{-1}\right)$ exiting the gully. a) The effect of catchment works in reducing the flow entering the gully head $(Q)$, where the control represents a $Q$ of 8.0 $\mathrm{m}^{3} \mathrm{~s}^{-1}$, with 'small', 'medium', 'large', and 'very-large' representing reducing $Q$ by $20 \%\left(6.4 \mathrm{~m}^{3} \mathrm{~s}^{-1}\right), 40 \%$ $\left(4.8 \mathrm{~m}^{3} \mathrm{~s}^{-1}\right), 60 \%\left(3.2 \mathrm{~m}^{3} \mathrm{~s}^{-1}\right)$, and $80 \%\left(1.6 \mathrm{~m}^{3} \mathrm{~s}^{-1}\right)$, respectively. b) The effect of increasing the vegetation cover $(V C)$ in the gully channel, where the control represents a $V C$ of $0 \%$, with 'small', 'medium', 'large', and 'very-large' representing $V C$ of $20 \%, 40 \%, 60 \%$, and $80 \%$, respectively. c) The effect of rock-capping the gully head, where the control represents the gully under normal conditions, and rock capped represents no erosion within the gully head.

resulted in the greatest reduction to $Q C(56-78 \%$, dependent on $J)$ exiting the gully. By contrast, the combination of reducing $Q$ and rock-capping resulted in the lowest reduction to $Q C$, where its effect on reducing $Q C$ was similar to the sole vegetation cover intervention at high $J$ values and less than the sole vegetation cover intervention at low $J$ values. The combination of all three interventions gave the greatest reduction of $Q C(65-82 \%$, dependent on $J$; Figure 4).

\section{DISCUSSION}

This study explored the use of MERGE (Roberts 2020) to guide on-ground decision making for gully management. Using MERGE we quantified the expected benefits of different interventions, and combinations of 
Prentice et al., Assessing performance of the MERGE model for simulating gully interventions

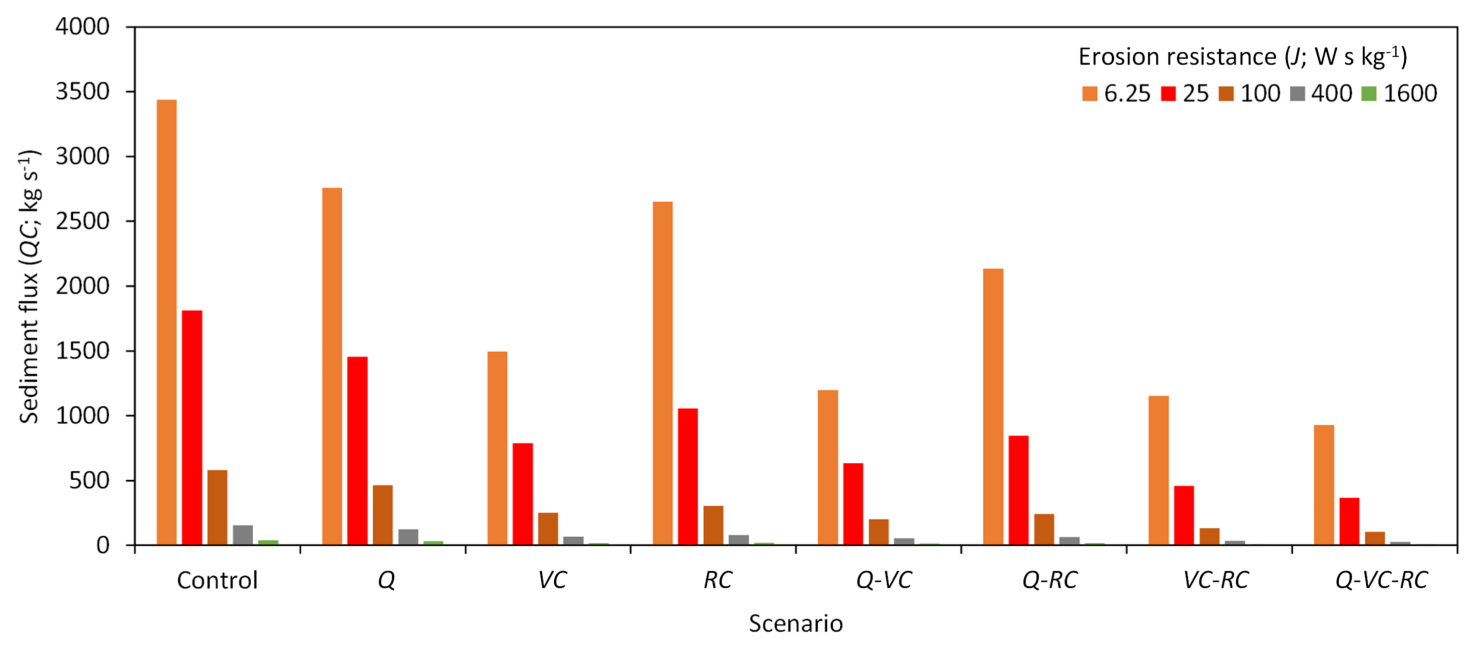

Figure 4. Comparing the effect of 'small' interventions to the flow entering at the gully head $(Q)$, 'small' interventions to vegetation cover $(V C)$ in the gully, and rock-capping the gully head $(R C)$-both in isolation and in combination-on the sediment flux $\left(Q C ; \mathrm{kg} \mathrm{s}^{-1}\right)$ exiting the gully. The control represents the gully under normal conditions, and interventions represent reducing $Q$, increasing $V C, R C$, reducing $Q$ and increasing $V C(Q-V C)$, reducing $Q$ and $R C(Q-R C)$, increasing $V C$ and $R C(V C-R C)$, and all three interventions combined $(Q-V C-R C)$.

interventions, providing actionable information to support gully management focused on downstream impacts. Each gully intervention performed markedly differently depending on gully conditions. Further, different interventions resulted in different rates of reduction (i.e., linear and non-linear) measured by $Q C$ exiting the gully. The benefits of combined interventions were not additive, illustrating how a process-based model can be used to assess potential outcomes within the typical management approach of integrating multiple interventions.

MERGE clearly demonstrated that identical types of interventions vary with gully conditions. For example, the effect of rock-capping on a highly erosive $(J=0)$ gully (c.f., a moderately erosive $[J=100]$ gully) would be diminished due to channel flux $(Q C)$ quickly achieving $C^{*}$ (i.e, the maximal sediment transport). As such, rock-capping in this scenario would have little effect in reducing $Q C$ at the gully exit, although localised benefits not captured by the model would be expected. Although there is some uncertainty in the $C^{*}$ value adopted in this study, our results are consistent with current gully rehabilitation practices where major engineering works to reshape gullies are often preferred in highly erosive sodic soils (Wilkinson et al. 2019). Moreover, when $J$ was sufficient to maintain concentrations below $C^{*}$, MERGE demonstrated the reduction in $Q C$ at the gully exit was significant. In addition, the relative reduction in $Q C$ was greatest at higher $J$ values, and the absolute reduction in $Q C$ was greatest at lower $J$ values. These results demonstrate the importance of considering both relative and absolute reductions when evaluating the effectiveness of different interventions (noting that cost-effective metrics commonly use \$/tonne).

MERGE demonstrated that a reduction in $Q$ resulted in a proportional linear reduction in $Q C$ exiting the gully, whereas in increase in vegetation cover resulted in a non-linear reduction in $Q C$ exiting the gully, with ever increasing vegetation cover producing reduced returns. The representation of vegetation cover in the model, however, can be considered conservative, as increased vegetation cover not only increases $n$, thus reducing $Q$, but also increases soil cohesion $(J)$, thus reducing erodibility; the later of which is not captured in the vegetation model (Roberts 2020). Therefore, further research is required to incorporate effects on $J$ within the vegetation model. Both $Q$ and vegetation cover interventions showed consistent proportional reductions in $Q C$ across all $J$ values considered, although improvements to the vegetation model may lead to different relationships. In contrast, rock-capping (c.f. the control) resulted in the relative reduction of $Q C$ to increase with $J$. This could be due to the effect of rock-capping being constrained to the gully head (the first metre at Fernvale Gully), unlike $Q$ and vegetation cover interventions, which apply along the full gully length.

MERGE demonstrated that the benefits of combined interventions were not additive. In Fernvale Gully, the most effective intervention to reduce $Q C$ exiting the gully would be a combination of catchment works to reduce $Q$, vegetating the gully channel, and rock-capping of the gully head. The combination of rock-capping 
and vegetation cover, however, produced a reduction in $Q C$ at the gully exit of between 56-78\% (c.f., 65$82 \%$, with all three interventions), indicating a reduction in $Q$ (via catchment works) in addition to these two interventions may produce limited returns.

The considerable variation in effectiveness (absolute, and-in the case of rock-capping-relative) of the interventions under different $J$ values highlights the importance of reducing the uncertainty of $J$. Knowledge of $J$ under different soil conditions, however, is lacking in the scientific literature. Therefore, in our study it has been necessary to consider a wide range of plausible values, leading to high uncertainty in the expected absolute reductions in $Q C$ exiting the gully, albeit with less uncertainty in the expected relative reductions. With $J$ being relatively unknown in the literature, we emphasise that future research should pursue the development of methods to quantify $J$. Such research might involve the use of historical records of erosion to constrain $J$ to narrow ranges within a site. Similar to $J, C^{*}$ is also not well quantified within the literature, and was observed to constrain the benefit of rock-capping interventions in particular. Although this behaviour is expected, there is high uncertainty around the $C^{*}$ value used. Confidence in the model performance, especially with low $J$ values, would be improved by reducing uncertainty in this parameter.

Natural resource management (NRM) professionals are faced with the difficult task in determining how best to invest and allocate their limited resources to a wide array of environmental challenges (e.g., Wilkinson et al. 2019). To achieve these challenges, each investment decision is typically stress tested and prioritised. MERGE provides NRM professionals with a tool to readily quantify the predicted volume of eroded sediments for a given site. This quantification provides decision makers with the ability to allocate a dollar value per tonne of sediment reduced, thus providing assurance to NRM professionals and stake-holders that the projects they invest in are both efficacious and cost-effective. As such, future use of the model should look to apply scenarios in a management sense, and include gullies with complex morphologies (i.e., dendritically shaped, and multiple heads), as well as a broader suite of interventions (e.g., such as porous check dams, and application of gypsum and fertilisers; Wilkinson et al. 2019, Bartley et al. 2020).

\section{ACKNOWLEDGEMENTS}

This project received funding from the Queensland Government through the Queensland Water Modelling Network.

\section{REFERENCES}

Bartley, R., Poesen, J., Wilkinson, S. \& Vanmaercke, M. (2020), 'A review of the magnitude and response times for sediment yield reductions following the rehabilitation of gullied landscapes', Earth Surface Processes and Landforms 45(13), 3250-3279.

Castillo, C. \& Gómez, J. (2016), 'A century of gully erosion research: Urgency, complexity and study approaches', Earth-Science Reviews 160, 300-319.

Cuddington, K., Fortin, M.-J., Gerber, L. R., Hastings, A., Liebhold, A., O'Connor, M. \& Ray, C. (2013), 'Process-based models are required to manage ecological systems in a changing world', Ecosphere 4(2), 112.

Hamilton, D. P., Salmaso, N. \& Paerl, H. W. (2016), 'Mitigating harmful cyanobacterial blooms: strategies for control of nitrogen and phosphorus loads', Aquatic Ecology 50(3), 351-366.

Prosser, I. P. (2018), 'Improving how gully erosion and river sediment transport processes are represented in Queensland catchment models'.

Roberts, M. E. (2019), The erosion of an ideal gully under steady state conditions, in '23rd International Conference on Modelling and Simulation', Canberra, Australia.

Roberts, M. E. (2020), 'MERGE: modelling erosion resistance for gully erosion - a process-based model of erosion from an idealised linear gully', Soil Research pp. 1-16.

Roberts, M. E., Burrows, R. M., Thwaites, R. N. \& Hamilton, D. P. (submitted), 'Modelling classical gullies a review'. Submitted May 2021.

Wilkinson, S., Hairsine, P., Brooks, A., Bartley, R., Hawdon, A., Pietsch, T., Shepherd, B. \& Austin, J. (2019), Gully and stream bank toolbox. a technical guide for the reef trust gully and stream bank erosion control program. 2nd edition, Technical report, Commonwealth of Australia. 